\title{
Surgical Results of Brain Metastasis from Lung Cancer -Prognostic Factors-
}

\author{
Yutaka IDE, Kazunari OKA, Hirohito TsuCHIMOCHI, \\ Tsuyomi Mizoguchi, Takeo FukUShIMA, Masamichi TomonaGA \\ and Takayuki SHIRAKUSA*
}

Department of Neurosurgery and *Second Department of Surgery, School of Medicine, Fukuoka University, Fukuoka

\begin{abstract}
Twenty-five patients receiving surgical treatment for brain metastasis from lung cancer were retrospectively studied to evaluate the prognostic factors for survival time. Twenty-two patients had died of respiratory distress by April, 1989. Favorable prognostic factors derived from the median survival time (MST) in these patients included; 1) resection of primary tumor (MST 10 months); 2) total or subtotal removal of metastatic tumor (MST 6.5 months); 3) adenocarcinoma (MST 13 months); 4) metachronous onset of brain metastasis (MST 12 months); 5) single metastasis (MST 8 months). These results suggest that therapy for the primary lung cancer is important before surgery for metastatic brain tumor.
\end{abstract}

Key words: lung cancer, brain metastasis, surgical treatment, prognosis

\section{Introduction}

Metastatic brain tumors account for approximately $13 \%$ of all brain tumors in Japan. ${ }^{21}$ Lung cancer is the main cause of metastatic brain tumors and the incidence continues to increase. Brain metastasis is the most important systemic relapse in lung cancer and adversely influences the long-term prognosis, ${ }^{1,10.111}$ so metastatic foci should be treated. The prognosis remains poor in spite of various therapeutic trials. An analysis of the 451 patients treated for primary lung cancer in the past 14 years in our institute showed both survival time and median survival time (MST) of patients with brain metastasis were significantly shorter than in cases without metastasis ( $p<$ 0.001 ): MST was 6.7 months for metastasis, and 14 months for nonmetastasis cases.

The favorable factors influencing the clinical outcome are significant in the treatment of metastatic cancer. We therefore analyzed retrospectively the surgical outcomes of brain metastases from lung cancer during the past 11 years.

Received June 1, 1989; Accepted October 18, 1989

\section{Materials and Methods}

From April, 1978, through March, 1989, 25 patients with primary lung cancer underwent intracranial surgery for brain metastasis. They comprised 19 males and six females with an age distribution of 38 73 years (mean 60 years). All patients received intracranial surgery before irradiation or chemotherapy because of acute neurological deterioration. Five patients were treated initially as cases of primary brain tumor. Surgical procedures were based on the guidelines of Black ${ }^{1)}$ and Yamashita. ${ }^{12)}$ Computed tomographic (CT) scanning was useful for the clinical diagnosis of brain metastasis. The final diagnosis was made by histological examination of surgical specimens. The criteria for evaluation of outcome were: survival time, average survival time (AST), and MST from intracranial surgery to death. A MST over 5 months was regarded as a favorable result. . $^{911,12)}$

The survival data in the 22 cases of deaths were analyzed using the Kaplan-Meier method. The chisquare test and Wilcoxon test were also used for comparisons. The prognostic indicators evaluated were: time before diagnosis; intracranial procedure; location and number of metastatic foci; resectability and 
histology of the primary tumor.

Time before diagnosis as brain metastasis: $\mathrm{Pa}-$ tients were divided into 2 groups, the synchronous onset group $(n=13$, presenting with brain metastases as an initial manifestation), and the metachronous onset group ( $\mathrm{n}=9$, brain metastases occurring after treatment for the primary tumor). The synchronous onset group was divided into a group with neurological deterioration $(\mathrm{n}=8$, treated surgically because of acute neurological deterioration after a diagnosis of primary lung cancer) and a group where the metastatic origin was difficult to determine $(\mathrm{n}=5$, no diagnosis of primary lung cancer was made before intracranial surgery).

Methods of intracranial surgery: Patients were divided into 2 groups, a total or subtotal removal group $(n=14)$ and a decompression group $(n=8)$. "Decompression" included internal decompression, burr-hole aspiration of a tumor cyst, ventricular drainage, and ventriculoperitoneal shunt.

Location and number of metastatic foci: These were supratentorial $(n=15)$ and infratentorial $(n=$ 7) metastases. Patients were classified as single metastasis $(n=19)$ and multiple metastases $(n=3)$.

Resectability of primary tumor: Patients were divided into a resectable group $(n=16)$ and a nonresectable group $(n=6)$. Patients in the nonresectable group were treated with irradiation and/or chemotherapy.

Histology of primary lung cancer: Histological types found were adenocarcinoma $(n=9)$, squamous cell carcinoma $(\mathrm{n}=8)$, and undifferentiated carcinoma $(n=5)$. Undifferentiated carcinoma included small cell carcinoma and large cell carcinoma.

\section{Results}

\section{Summary of clinical data}

At time of analysis, of the 25 patients treated for brain metastasis, 22 patients had already died. Metastasis was not diagnosed prior to intracranial operative procedures in five patients. The correct diagnosis was $80 \%$ preoperatively.

Histology, sex, and metastatic onset: Table 1 summarizes the histology, sex, and metastatic onset. There were 10 patients with adenocarcinoma (40\%), nine with squamous cell carcinoma $(36 \%)$, and six with undifferentiated carcinoma (24\%). The relationship between histology and sex was not statistically significant, but there was a significant relationship between onset of metastasis and histology $(p<0.05)$.

Location and number of metastatic foci: There were 18 patients with supratentorial metastasis and seven with infratentorial metastasis. Supratentorial metastasis occurred in the frontal lobe in 12 cases and multiple metastases in two. All seven infratentorial metastasis cases occurred in the cerebellar hemisphere, and only one with multiple metastases. No case presented metastasis in both supra- and infratentorial regions.

Clinical manifestations on diagnosis: Twenty patients had headache due to increased intracranial pressure, 13 focal neurological signs (eight paresis and five cerebellar ataxia), two had mental deterioration, one loss of consciousness, and one no neurological deficits. None presented with seizure or intracranial hemorrhage from metastatic tumor.

Surgical procedure and outcome: Metastatic tumor was totally removed in 14 patients, subtotally in three, and eight received internal decompression. All cases of total and subtotal removal had a single metastasis. Of the eight decompression cases, five had a single and three multiple metastasis. The five single metastasis cases underwent decompression only because of poor general condition. The postoperative neurological status improved in 20 patients $(80 \%)$ and was unchanged in five. No neurological deterioration, cerebrospinal fluid dissemination of tumor cells, or local metastatic recurrence were found after intracranial surgery.

Primary tumor management after intracranial surgery: Nine synchronous onset patients underwent surgery and postoperative chemoradiotherapy, while six received chemoradiotherapy only for the primary

Table 1 Histology, sex, and mode of brain metastatic onset

$\begin{array}{ccccc}\text { Histology } & \begin{array}{c}\text { Male } \\ (\mathrm{n}=19)\end{array} & \begin{array}{c}\text { Female } \\ (\mathrm{n}=6)\end{array} & \begin{array}{c}\text { Synchronous } \\ \text { onset } \\ (\mathrm{n}=15)\end{array} & \begin{array}{c}\text { Metachronous } \\ \text { onset } \\ (\mathrm{n}=10)\end{array} \\ \begin{array}{l}\text { Squamous cell carcinoma }(\mathrm{n}=9) \\ \text { Adenocarcinoma }(\mathrm{n}=10)\end{array} & 8 & 1 & 4 & 5 \\ \text { Undifferentiated carcinoma }(\mathrm{n}=6) & 5 & 5 & 5 & 5 \\ \end{array}$

Undifferentiated carcinoma tended to occur in synchronous onset of metastasis $(p<0.05)$. 
lung cancer.

\section{Survival time}

In the 22 patients who died by April, 1989, the period from surgery for lung cancer to death ranged from 1 to 48 months (AST 13.2 months, overall MST 8 months), and from surgery for brain metastasis to death was 1-28 months (AST 9 months, MST 6.5 months) (Fig. 1A). The MST of 6.5 months is slightly longer than the 5 months intended and is regarded as a favorable result.

Survival time and sex: The survival time in 16 males ranged from 1 to 17 months (AST 6.4 months, MST 3 months). On the other hand, in six females it was 8-28 months (AST 17.7 months, MST 19 months) (Fig. 1B). Females had a significantly longer survival $(p<0.05)$.

Mode of metastatic onset (Fig. 1C): In the metachronous onset group, the period between resection of primary lung cancer and brain metastasis ranged from 1 to 32 months (mean 11 months), between surgery for brain metastasis and death ranged from 1 to 28 months (AST 11 months, MST 12 months), and between diagnosis of primary lung cancer and death 3-48 months (AST 23 months, MST 19 months).

The period between surgery for brain metastasis and death in the synchronous onset group ranged from 1 to 24 months (AST 7.6 months, MST 5 months). In patients with neurological deterioration, the survival period ranged from 1 to 13 months (AST 4.3 months, MST 3.5 months), and in the group with delayed metastasis diagnosis from 2 to 24 months (AST 13 months, MST 8 months).

The metachronous onset group had a longer MST, but the statistical difference was not significant. The survival time of the metachronous onset group, however, was significantly longer than in the synchronous group with neurological deterioration $(\mathrm{p}<$ 0.05 ).

Intracranial procedure (Fig. 1D): The survival period of the total or subtotal removal group ranged from 1 to 28 months (AST 10 months, MST 6.5 months), and of the decompression group from 1 to 17 months (AST 8 months, MST 5 months). The total or subtotal removal group had a longer MST, but the difference was not statistically significant.

Location and number of brain metastasis: The survival period of patients with supratentorial metastasis ranged from 1 to 28 months (AST 7.8 months, MST 5 months), and with infratentorial metastasis from 1 to 23 months (AST 10 months, MST 5 months). The difference was not statistically significant.
The survival period of patients with a single metastasis ranged from 1 to 28 months (AST 9.9 months, MST 8 months), and with multiple metastases ranged from 1 to 8 months (AST 3.7 months, MST 2 months). Single metastasis patients had a significantly longer survival time $(p<0.05)$.

Resectability of primary lung cancer: The survival time of patients with resectable lung tumor ranged from 1 to 28 months (AST 10 months, MST 10 months) and with nonresectable tumor from 1 to 24 months (AST 6.7 months, MST 2.5 months) (Fig. $1 \mathrm{E})$. The patients with resectable lung cancer had a significantly longer survival time $(\mathrm{p}<0.05)$.

In the synchronous onset group, the survival period of the resectable cases ranged from 1 to 23 months (AST 8.4 months, MST 5 months) and that of the nonresectable cases from 1 to 24 months (AST 6.7 months, MST 2.5 months). This difference was not statistically significant.

Differences in histology (Fig. 1F): For adenocarcinoma, the survival period ranged from 2 to 24 months (AST 12.7 months, MST 13 months); in squamous cell carcinomas from 1 to 28 months (AST 8.5 months, MST 3.5 months), and in undifferentiated carcinomas from 1 to 8 months (AST 3.4 months, MST 2 months). Patients with undifferentiated carcinoma had a significantly shorter survival time $(\mathrm{p}<0.05)$.

\section{Survival cases}

Three patients (adenocarcinoma, squamous cell carcinoma, and undifferentiated carcinoma in one case each) survived at March, 1989. The squamous cell carcinoma and undifferentiated carcinoma patients in the preoperatively undiagnosed group have survived for 2 months after intracranial surgery.

An adenocarcinoma patient has survived for 62 months after primary lung cancer diagnosis and is considered completely cured. This 64-year-old male underwent right upper lobectomy for primary lung cancer (Fig. 2 left) in March, 1984 and received postoperative chemoradiotherapy. He was well for approximately 30 months afterwards. However, he gradually developed headaches, amnesia, and aphasia in August, 1986. A postcontrast CT scan showed a left frontal lobe tumor (Fig. 2 right). The brain tumor was totally removed. Histological examination revealed a brain metastasis from the lung cancer. He has completely recovered.

\section{Discussion}

The factors reported to affect the final outcome in lung cancer associated with brain metastasis are: 

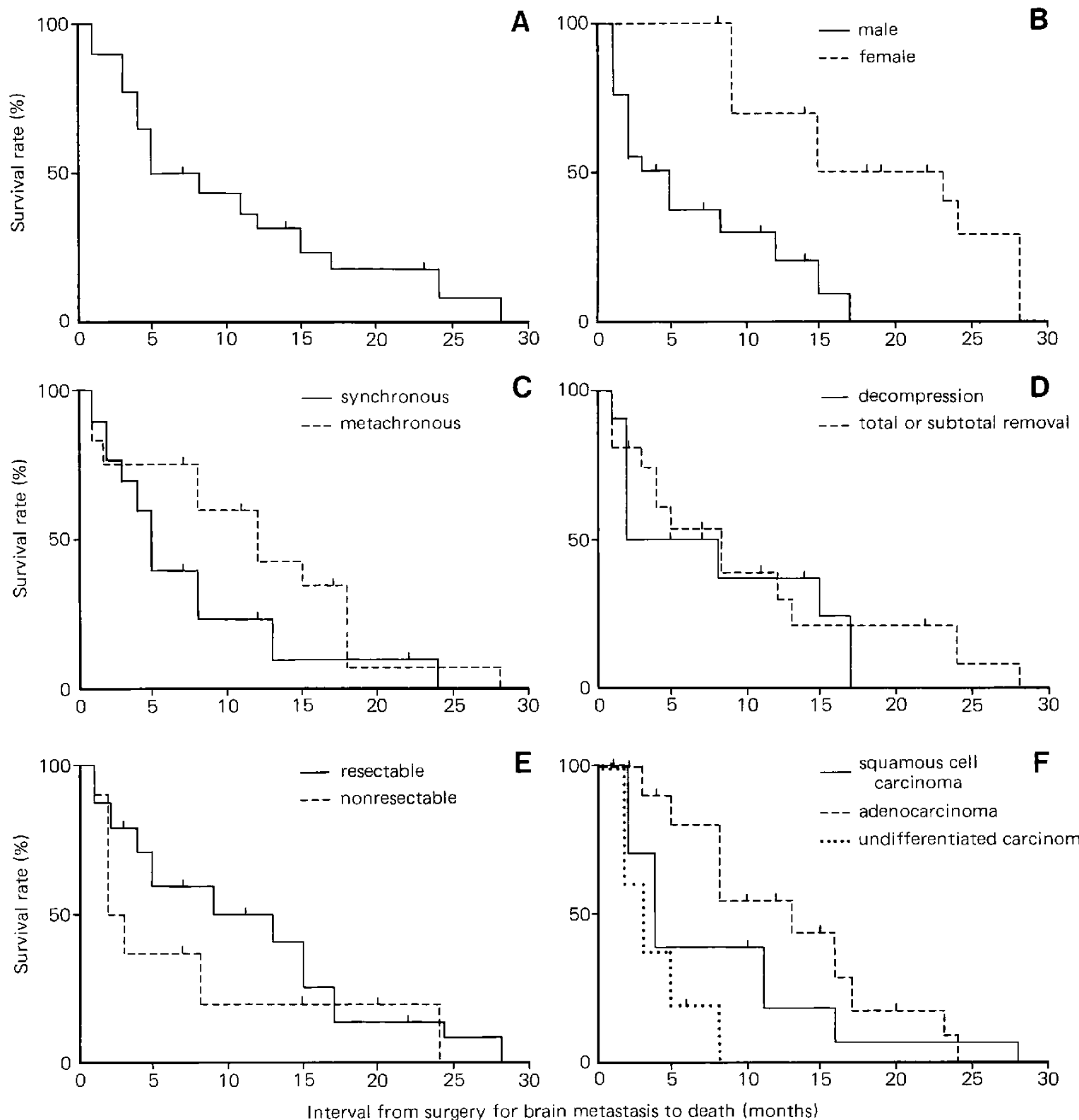

Fig. 1 A: Survival times for 22 patients receiving surgery for brain metastasis from lung cancer between 1978 and 1989. B: Relationship between survival time and sex. Male MST was 3 months $(\mathrm{n}=16$ ) and female MST was 19 months $(n=6)$, demonstrating a significant difference $(\mathrm{p}<0.05)$. C: Survival time and mode of brain metastatic onset. MST in the synchronous onset group $(\mathrm{n}=13$ ) was shorter than in the metachronous onset group $(n=9)$, but without significant difference. $D$ : Survival time and surgical procedure for metastatic brain tumor. There was no significant difference between total or subtotal removal $(n=14)$ and decompression $(n=8)$. E: Survival time and resectability of primary lung cancer. The resectable group $(n=16)$ and nonresectable group $(n=6)$ differed significantly $(p<0.05)$. F: Survival time and histological classification of lung cancer. There was a significant difference between adenocarcinoma $(n=9)$ and undifferentiated carcinoma $(\mathrm{n}=5)(\mathrm{p}<0.05)$, but not between adenocarcinoma and squamous cell carcinoma $(n=8)$. 

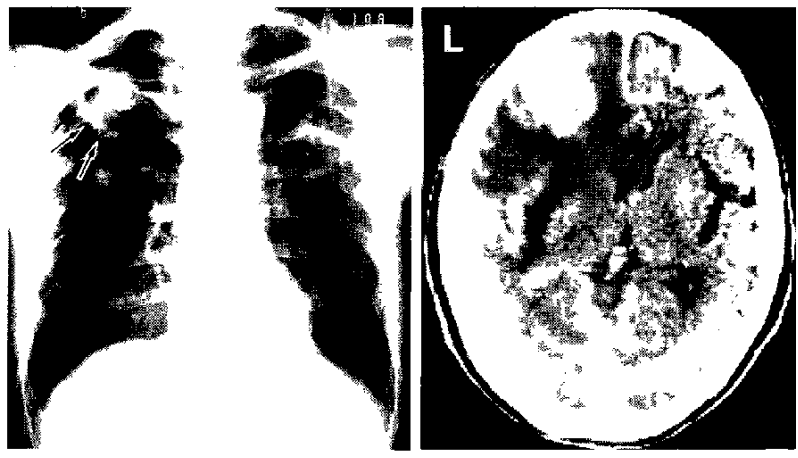

Fig. 2 A long survival case with surgery for both lung cancer and brain metastasis. left: Preoperative chest $x$-ray film revealing an abnormal shadow in the right upper lobe (arrows). right: Postcontrast CT scan 2.5 years after the operation for lung cancer, demonstrating an enhanced brain tumor with perifocal edema in the left frontal lobe.

period before diagnosis of brain metastasis ${ }^{3,7,9)}$; method of treatment ${ }^{9.11,12)}$; invasion of primary tumor according to the tumor, metastasis, nodes (TMN) classification ${ }^{4,8)}$; status of primary tumor ${ }^{6,99}$; histological classification of primary tumor ${ }^{4,6,7,10)}$; number of metastases ${ }^{6,10,12)}$; and clinical manifestation of the metastatic brain tumor. ${ }^{12)}$

The relationship between these factors and longterm results was studied in patients receiving intracranial surgery for brain metastasis. The overall MST was 6.5 months after surgery. However, this is not superior to other survivals reported earlier such as $3.1,{ }^{12)} 5.7,{ }^{7)} 6,{ }^{5)}$ and 14 months. ${ }^{9)}$ No significant difference in survival time was found between the synchronous and the metachronous groups. Hoshino et al. ${ }^{37}$ reported a similar result. However, Sundaresan et al. ${ }^{\text {9) }}$ found that the survival time in the metachronous group was longer. This may be due to the different patient groups in these studies.

Eighty percent of patients with primary lung cancer were accurately diagnosed as having brain metastasis before intracranial surgery in our cases. Accurate diagnosis ranges from approximately 76 to $79.2 \%$ in the literature. ${ }^{5,6)}$ This suggests the differential diagnosis of brain tumors requires more investigation.

There was no significant difference in survival time between the total or subtotal removal and the decompression groups. Previous investigation of the survival time in brain metastasis and therapeutic method show that: 1) decompression alone is ineffective, ${ }^{101} 2$ ) the survival period depends on the general condition at admission more than on treatment, ${ }^{12}$ and 3) multidisciplinary treatment is effective for primary cancer with brain metastasis. ${ }^{5,8,10)}$ Intracranial surgery is generally chosen for patients in good general condition.

Therapeutic results for multiple metastases were inferior to those for single metastasis. We believe that surgery for multiple brain metastases is inappropriate.

The group with rapid neurological deterioration achieved very poor results due to aggravated clinical features. Neurosurgical treatment may be ineffective in such cases. However, Yamashita ${ }^{12)}$ reported intracranial surgery was useful in patients with increased intracranial pressure.

The therapeutic results in brain metastasis are influenced by the primary tumor treatment..$^{3,6,7,9,12)}$ The survival time for patients with resected lung cancer is apparently superior to those not resected in our cases. The metachronous onset group has a longer survival time, but the statistical difference was not significant.

Previous intracranial surgery series show $47 \%{ }^{51}$ to over $70 \%{ }^{8}$ of patients die of respiratory failure. Our clinical outcomes were similar to these earlier cases. Thus management of the primary tumor is very important after intracranial surgery. ${ }^{6,9)}$

\section{References}

1) Black P: Brain metastasis: Current status recommended guidelines for management. Neurosurgery 5: 617631,1979

2) The Committee of Brain Tumor Registry in Japan: Brain Tumor Registry in Japan, vol 6. 1987, $175 \mathrm{pp}$

3) Hoshino T, Satoh T, Fujita T, Moriya H, Kageyama K, Suzuki S, Kanno J, Satoh T, Hada K, Yaginuma $\mathrm{K}$, Muraoka $\mathrm{H}$, Hoshi K, Kimura K: Clinical findings and therapeutic results of brain metastasis from lung cancer. Fukushima Igaku Zasshi 36: 127-131, 1986 (in Japanese)

4) Ikegami H, Nakamura S, Hourai T, Mori S, Inoue T, Tateishi R, Doi O: Characteristics and treatment of CNS metastasis in lung cancer. Gan No Rinsho 30: 981-986, 1984 (in Japanese)

5) Kitaoka K, Abe H, Nakagawa $Y$, Satoh M, Itoh T, Kawamoto $T$ : Assessment of surgery for solitary brain metastasis from lung cancer. Neurol Med Chir (Tokyo) 28: 259-264, 1988 (in Japanese)

6) Kobayashi T, Kida Y, Yoshida J, Shibuya N, Inoue I, Kageyama N, Ohta K, Nishimura M: Diagnosis and treatment of brain metastasis of lung cancer in CT era. Correlation to histological types. Neurol Med Chir (Tokyo) 22: 446-452, 1982 (in Japanese)

7) Nishimura $\mathrm{H}$ : Brain metastasis in lung cancer. Analysis of 60 cases. Kurume Med $J$ 49: 221-236, 
1986 (in Japanese)

8) Nomura K, Watanabe $T$, Nakamura O, Shibui S, Takakura K: Studies on prognosis of metastatic brain tumor from lung cancer with respect to its stages. No Shinkei Geka 13: 159-165, 1985 (in Japanese)

9) Sundaresan N, Galicich JH, Beattie EJ: Surgical treatment of brain metastasis from lung cancer. $J$ Neurosurg 58: 666-671, 1983

10) Takakura K: Multidisciplinary treatment for metastatic brain tumor. Gan No Rinsho 26: 685-689, 1980 (in Japanese)

11) Takeda F, Kawashima Y, Fujii T, Uki J: Metastatic brain tumors. Results of the treatment of multiple modalities. Neurol Med Chir (Tokyo) 21: 1033-1040, 1981 (in Japanese)

12) Yamashita J: Surgical treatment of brain metastases. Gan No Rinsho 30: 1010-1016, 1984 (in Japanese)

Address reprint requests to: Y. Ide, M.D., Department of Neurosurgery, School of Medicine, Fukuoka University, 7-45-1 Nanakuma, Johnan-ku, Fukuoka 81401 , Japan. 